\title{
Website Structure Optimization Model Based on Ant Colony System and Local Search
}

\author{
Harpreet Singh \\ Guru Nanak Dev University, Amritsar, India \\ harpreet99.nitj@gmail.com \\ Parminder Kaur \\ Guru Nanak Dev University, Amritsar, India \\ parminderkaur@yahoo.com
}

\begin{abstract}
The unabated growth of the World Wide Web in the last decade and the increasing size of the websites have resulted in significant amount of research activity to improve the link structure of the websites. A website can be considered as a directed graph with webpages as nodes and hyperlinks as edges referred to as the Webgraph. Website structure optimization or reorganization is also considered as a graph optimization problem. The researchers have developed few models to optimize the website link structure. It is observed that the heuristic and mathematical models cannot optimize webgraphs of large size and are also time consuming. This paper presents an Ant Colony and local search based hybrid metaheuristic model for the newly emerged website structure optimization (WSO) problem. The developed hybrid model is also compared with Ant colony method and it is observed that the model performs better than Ant Colony System based approach.
\end{abstract}

Index Terms- Web Graph; Optimization; Ant Colony System; Local Search

\section{INTRODUCTION}

With rapid growth of the World Wide Web and Internet, websites are becoming a competitive tool for businesses. The development of well- structured website is a major challenge in the E-business industry. The task of designing an effective website structure is not a trivial one [4]. A number of high profile site failures have been there due to poor link structure design of the websites [13]. Users facing difficulty in getting to the target pages are very likely to leave a website even if the information offered by the website is of very high quality [13].

A user during a visit to a website would like to get to the required information as quickly as possible[24]. An easily navigable website and less time and effort in finding the required webpage adds to the user satisfaction [8]. For a commercial website, good link structure and easy navigation can encourage online purchases [17], whereas an ill- structured website would not be able hold on to its customers [21]. Web usage data stored in weblogs at web servers are used to improve the structure improvement [7].

Adaptive websites [14] are sites that repeatedly improve their link structure by learning from visitor access patterns. Website structure reorganization can be considered as a special graph optimization problem.
Website users look for browsing experience which requires the website to be efficient. Efficient website means that the users should reach their target page in the website with just few clicks. Perkowitz and Etzioni [15] defined that the approaches to improve browsing efficiency of a website can be classified into two types: Customization methods and Transformation methods. Customization means aiding a user by providing guidance. In customization, mostly additional index pages are created which contain links to other pages according to previous users visits. Transformation involves reorganizing the links among web pages. It involves modifying the Web site structure to ease the navigation for all users. The advantages of reorganizing the Web site structure include improved Web usage mining efficiency and lower memory requirement for storing the pages and the user browsing history. In this paper, a transformation based method is presented. The remainder of the paper is organized as follows. Section 2 presents the related work. Section 3 presents the formulation of model. Section 4 presents the proposed technique. Section 5 presents experimental results. Section 6 concludes the work.

\section{RELATED WORK}

Fu et al. [5] have proposed the method in which the structure of the website evolves over time. The proposed method uses operations such as merging of webpages and removal of links. Lin [10] developed a 0-1 programming model for reorganizing Web sites based on user browsing data. A method to optimize the website link structure for E- supermarket websites is developed by Wang, Y. et al. [22]. The problem is mapped onto Hopfield networks [16]. This model works by reducing the overall weighted distance between webpages. Saremi et al. [18] developed a method in which the Website Structure Optimization problem is modeled as quadratic assignment (QAP) [12]type problem. Ant colony meta-heuristic technique is employed to solve the problem. Lin and Tseng [11] developed a model based on ant colony [2] system for website reorganization. The method is divided into two stages. In the first stage, the Ant Colony based method is applied to find a spanning tree and in the second stage, a 
simple deterministic procedure is applied to form the optimal web structure. Chen and Ryu [1] have developed a mathematical programming (MP) model that assists the user navigation on a website with minimal changes to its current structure. Yin and Guo [23] have presented a metaheuristic based on Tabu search [6] approach for obtaining an optimal website structure. Website Structure Optimization problem is modeled as quadratic assignment problem and Tabu search meta-heuristic technique is used to solve the problem. This article presents a hybrid model based on Ant Colony and Local search [20] to optimize the link structure. In this paper, a website is regarded as a directed graph with nodes representing webpages and edges represent links among the webpages as shown in figure 1. Link matrix [22] of a webgraph represents the link structure of the website. The elements of a link matrix $\mathrm{L}$ can have binary values. In link matrix $\mathrm{L}, \mathrm{L}_{i, j}=1$ denotes that there exists a hyperlink from node $\mathrm{i}$ to node $\mathrm{j}$ : otherwise link does not exist. Figure 2 represents the link structure of webgraph of figure 1 .

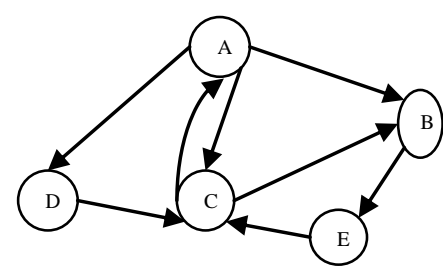

Fig. 1. Hyperlink graph example

$\begin{array}{cccccc}\text { Nodes } & \text { A } & \text { B } & \text { C } & \text { D } & \text { E } \\ \text { A } & 0 & 1 & 1 & 1 & 0 \\ \text { B } & 0 & 0 & 0 & 0 & 1 \\ \text { C } & 1 & 1 & 0 & 0 & 0 \\ \text { D } & 0 & 0 & 1 & 0 & 0 \\ \text { E } & 0 & 0 & 1 & 0 & 0\end{array}$

Fig. 2. Link matrix L of web graph of figure 1

\section{PROBLEM FORMULATION}

A website is considered as a directed graph with nodes representing webpages and edges represent links among the webpages. Suppose there are $\mathrm{N}$ webpages $\left\{v_{i} \mid i \in\right.$ $[1, N]\}$ to be organized in the website structure. Here $v_{l}$ represents the home page. Every link has a weight denoted by the symbol $\mathrm{f}_{\mathrm{ij}}$, it specifies the access frequency of the link from node $i$ to node $j$. Access frequency corresponds to the number of visitors moving from page $\mathrm{i}$ to page $\mathrm{j}$. It can be obtained easily from web usage [19] data stored at web server logs. To optimize the model, the weights of the links are generated by dividing the access frequency of each link with frequency sum of all the links. This process is also called normalization [10]. Hence the weight of every link is a number between 0 and 1. The goal function maximizes the frequency summation of all the links with certain constraints. This model also follows three constraints which are described below.
Out-degree constraint: It specifies that the outdegree of a node i.e. the number of outward hyperlinks from a webpage should not be more than a specified limit.

Depth or level constraint: This constraint specifies that the length of the shortest paths from home page to other webpages is limited by a constraint.

Connectivity constraint: Any webpage in the link structure should be accessible from the home page node.

The formulation for the WSO problem is described below.

$$
\begin{aligned}
& \text { Maximize } \sum_{(i, j) \in E} f_{i j} x_{i j} \\
& \text { Subject to } \sum_{v_{j} \in A\left(v_{i}\right)} x_{i j} \leq d \\
& \sum_{i}^{n} x_{i j} \geq 1 i \in(2, \ldots n) \\
& \sum_{j}^{n} x_{1 j} \geq 1 \\
& \lambda_{i} \leq l, \forall v_{i} \in V \\
& x_{i j} \in\{0,1\}
\end{aligned}
$$

Here, $x_{i j}$ is a binary variable denoting whether link $(i, j)$ exist in the resultant webgraph. Constraint (2) specifies the outdegree constraint where $\mathrm{A}\left(\mathrm{v}_{\mathrm{i}}\right)$ represents set of succeeding nodes of $\mathrm{v}_{\mathrm{i}}$ and $\mathrm{d}$ represents the maximum outdegree of any webpage .Constraint (3) signifies that the each node should be pointed to by some other node, except the root node to keep the webgraph connected. Constraint (4) indicates that the home page should point to some other node. Constraint (5), where $\lambda_{i}$ represents the level of node $v_{i}$, requires that all the node levels be smaller than a predetermined depth $l$. Constraint (6) denotes that $\mathrm{x}_{\mathrm{ij}}$ is an zero- one variable. The final output structure is in the form of link matrix.

\section{ANT COLONY SYSTEM AND LOCAL SEARCH BASED HYBRID APPROACH FOR WSO}

The proposed methodology is divided into two stages. First, the ant colony based algorithm is applied to find the initial improved website link structure and then the resultant website structure is used as initial solution for the local search [6] algorithm to further improve the solution.

\section{A. Stage 1: Generation of solution subgraph}

In the first stage, the Ant Colony based approach proposed by Lin and Tseng [11] is applied to find the initial optimal link structure. In this method, the m numbers of ants are used one by one to generate the initial solution. This stage is further divided into two steps as described below.

Step 1

In this step, the Ant Colony System (ACS) [3] based approach is used to generate a spanning tree satisfying the outdegree and required level constraints. In this approach, Ant colony algorithm chooses edges repeatedly in the construction of the spanning tree. The ant chooses a single edge every time to develop the tree. The ants select edges from a set of candidate edges. Candidate edge set consists of edges having starting node in the 
constructing tree and an ending node that does not belong to the constructing tree.

Suppose $\mathrm{T}$ denotes the tree being constructed. The candidate edge set is denoted by $\mathrm{C}=\left\{(\mathrm{i}, \mathrm{j}) \mid \mathrm{v}_{\mathrm{i}} \in \mathrm{T}, \mathrm{v}_{\mathrm{j}} \notin\right.$ $\mathrm{T}\}$. The home page node is represented by $\mathrm{v}_{1}$ and is added to the empty tree $\mathrm{T}$ in the beginning. Initially, the edges adjacent to $\mathrm{v}_{1}$ are added to $\mathrm{C}$. The ant picks an edge from $\mathrm{C}$ according to the following transition rule. If a random number $r \leq r_{0}$, where $r_{0}$ is a predefined parameter between $[0,1]$ then the ant chooses edge $(\mathrm{x}, \mathrm{y})$ according to the following rule.

$$
f_{x y}=\max _{(i, j) \in c}\left\{f_{\mathrm{ij}}\right\},
$$

here $f_{i j}$ is the weight of edge from node $i$ to $j$

If $r>r_{0}$, then an edge is randomly selected from $C$ according to the following probability distribution

$$
p_{x y}= \begin{cases}\frac{\tau(x, y)}{\sum_{(i, j) \epsilon c} \tau(i, j)}, & \text { if }(x, y) \in C, \\ 0, & \text { otherwise }\end{cases}
$$

The above mentioned probability distribution allows the model to simulate the exploration behavior of the ants. If $\mathrm{r} \leq \mathrm{r}_{0}$, then the next link is chosen according to the edge weight value as mentioned above. The value of the parameter $f_{i j}$ specifies the weight of the edge from node $i$ to node $\mathrm{j}$. It corresponds to the number of visitors moving from page $\mathrm{i}$ to page $\mathrm{j}$ and it can be obtained easily from web server logs. The links that goes out from $\mathrm{v}_{\mathrm{y}}$ are added to $\mathrm{C}$ after edge $(\mathrm{x}, \mathrm{y})$ and node $\mathrm{v}_{\mathrm{y}}$ are added to constructing tree $\mathrm{T}$. Then the outdegree of $V_{x}$ and the depth of $V_{y}$ are analysed. If the degree of $V_{x}$ equals the specified limit then all out going links that leave $V_{x}$ are removed from C. Similarly, if the level of $V_{y}$ equals the specified limit then all edges that leave $V_{y}$ are ruled out. The tree satisfies the degree and level constraints with these steps. The construction of spanning tree is ended when the set of candidate links and nodes becomes null. Step 2

When an ant generates a spanning tree then second step is started. The edge with the largest frequency is consecutively selected from remaining edges to add to the constructing tree to form a subgraph that satisfies the specified level and degree constraints. Then, the pheromones on the edges of the obtained subgraph are revised by the following local updating rule.

$$
\tau_{i j}=(1-\rho) \tau_{i j}+\rho \Delta \mathrm{v}
$$

Here $\tau_{i j}$ denotes the pheromone [3] level on edge (i,j). The parameter denoted by symbol $\rho$ represents the local pheromone evaporation rate which lies between 0 and 1 , and the symbol $\Delta \mathrm{v}$ represents the pheromone variation, in the proposed method, it is set to initial pheromone level.

Every ant generates a solution subgraph that satisfies the required constraints and the best link structure out of $\mathrm{m}$ solutions generated by the $\mathrm{m}$ ants is passed to local search stage.

\section{B. Stage 2: Local search application}

The website structure obtained from the ant colony method act as the initial solution in the local search [9] method. The local search is applied on the structure repeatedly to find if the objective can be improved. The solution formation of the local search employs different neighborhood than ACS which increases the probability of getting an improved website structure.

\section{Initialization}

In website structure improvement problem, the decision variables have value of either 0 or 1 and candidate solutions are represented by a matrix having 0 or 1 as elements. As described in the previous section the webgraph structure generated by the ACS is taken as initial solution.

\section{Neighborhood structures}

The operations which are used to alter the website structure are link deletion and link insertion. These operations are used to generate the neighborhood structures to analyze if some structure improves the objective function.

First a candidate edge list is generated having only edges which exist in the original link structure but are excluded from the initial structure generated by the ant colony system. Every link in the candidate list has the source and destination nodes in the constructing tree. An edge from the candidate list is taken and inserted in the website structure. This insertion is also accompanied by an edge deletion operation so that the outward degree constraint is not violated. The edge to be deleted should be the edge of the lowest weight originating from a source webpage. Suppose a link with source node a and destination node $\mathrm{b}$ is picked from the candidate list for insertion, then out of all the outgoing edges from node a, the edge with the lowest weight should be removed to maintain the specified outdegree. In this approach, a link insertion move should be made only if the weight of the edge to be inserted is greater than the edge to be removed. In the link deletion and insertion operations, the connectedness of the structure should remain intact. The level constraint of the nodes should also be maintained during the neighborhood generation. Suppose the deletion of link from $x$ to $\mathrm{z}$ makes the level of node $\mathrm{z}$ more than the maximum allowed level then this deletion operation should be cancelled.

Memory structures

The proposed local search uses a memory structure named insert tabu list to boost the search efficiency of the algorithm. Our method uses tabu list to respond to link delete operations. The insert tabu list contains the links which cannot be inserted in the future moves. When a link is removed from the website structure then that link is added to the insert tabu list so that the link is not added in future steps.

The procedure for the local search is given below:

Procedure Local_search
Begin
Initialization of memory structures
Structure generate by ACS is taken as initial
Repeat
Generate neighbors by link deletion and link
insertion operations
Evaluate the neighborhood link structures


Select the best admissible solution among the neighborhood

Update tabu list

if the best-found solution is better than the current structure then the current solution is replaced by the best found structure until stopping criteria is satisfied end

Return the overall best solution

\section{Updation of pheromone levels}

The global updating rule (10) is applied to update the pheromones on the edges of the best link structure obtained after the local search step.

$$
\tau_{\mathrm{ij}}=(1-\alpha) \tau_{\mathrm{ij}}+\alpha \mathrm{Z}_{\mathrm{gb}}
$$

where parameter $\alpha$ denotes the global pheromone evaporation rate and lies between 0 and 1 , and

$Z_{g b}=\left\{\begin{array}{lr}S_{g b}, \text { if edge }(i, j) \in \text { the best structure so far } \\ \tau_{i j}, \text { otherwise, }\end{array}\right.$

Here $S_{g b}$ represents the objective function value of global best solution. The pheromone level is updated if an edge belongs to the global best structure so far.

\section{Example of link deletion and insert operation}

This subsection gives an example of a move during local search to generate a neighborhood solution. Figure 3 shows a part of a webgraph with edge frequencies normalized. Suppose the outdegree allowed is two and the candidate list of local search phase contains the edge from $d$ to e with weight 0.076 . Then the edge $d$ to $b$ is deleted and edge $\mathrm{d}$ to e is added. It can be observed in figure 4 . that the added edge has more weight than the deleted edge hence it would increase the objective function.

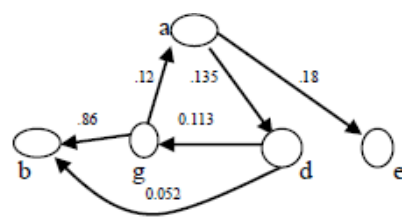

Fig. 3. A subgraph with normalized edge frequencies

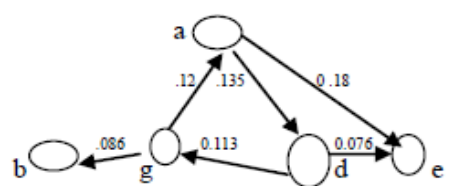

Fig. 4. Subgraph after edge deletion and insertion operation

\section{E. Pseudocode}

Pseudocode for the hybrid algorithm is given below:

Procedure ACS_local
Initialize memory structures
Begin

$$
\begin{aligned}
& \text { loop } \\
& \quad G=\text { Null, C=Null, } S^{g b}=\text { Null; } \\
& \text { // stage } 1 \text { starts }
\end{aligned}
$$$$
\text { While (the ending condition is not met) } / \text { main }
$$

For ant $k=1, \ldots, m$

$$
\begin{aligned}
& \quad \\
& T_{k}=\text { Null }, G_{k}=\text { Null; } \\
& T_{k}=T_{k}+v_{1} ; \\
& C=C+(1, j), \forall(1, j) \in G ;
\end{aligned}
$$

// the construction of the spanning tree starts While $(C \neq \varnothing)$

I

If $\left(r \leq r_{0}\right)$

Choose ( $x, y)$ according to equation (7)

Else

Choose ( $x, y)$ according to equation (8)

$T=T+(x, y)+v_{y}$

$C=C-(i, y), \forall(i, y) \in C$;

$C=C+(y, j), \forall(y, j) \in G$;

Determine the degree of $v_{x}$ and level of $v_{y}$; If ( the degree of $v_{x}=d$ )

$C=C-(x, j), \forall(r, j) \in C$;

If (the level of $v_{y}=l$ )

$C=C-(y, j), \forall(y, j) \in C$;

End \} // the spanning tree is constructed $G_{k}=T_{k}$;

$C=E-G_{k}$;

// step 2 of stage 1

While $(C \neq \varnothing)$

Find $(x, y), f_{x y}=\max _{(i, j) \in c}\left\{f_{i j}\right\}$

If (the degree of $v_{r}<d_{r}$ )

$G_{k}=G_{k}+(x, y)$,

$d_{x}=d_{x}+1$;

Else

$C=C-(x, y)$;

End // Step 2 of stage 1 ended

If $F(G)<F\left(G_{k}\right)$

$G=G_{k}$;

Local pheromone update using equation (9)

End // Stage 1 ends

\} // for loop ends

$C=E-G$;

// Here C denotes candidate edge set for local search

// stage 2 starts

Take initial solution $S=G$, and set $S^{l b}=S$

// local search loop starts below

While (termination condition not met) do

I

//apply Link insertion deletion operators to $S$

$N(S)=$ Generate_Neighborhood_Solutions $(S)$

Evaluate the solutions in $N(S)$;

Select the best solution among the neighborhood $N(S)$;

// updation of tabu list

Update the Memory Structures

//the best-found solution $x^{\text {lb }}$ is stored

if $\left(F(S)>F\left(S^{l b}\right)\right)$

$$
S^{l b}=S \text {; }
$$

End

) // the local search loop is ended

if $\left(F\left(S^{l b}\right)>F\left(S^{g b}\right)\right)$

$S^{g b}=S^{l b} / /$ the best-found solution $S^{g b}$ is stored

End

Global_pheromone update using equation (10)

\} // End of main while loop

End 
Here parameter $\mathrm{T}_{\mathrm{k}}$ denotes the spanning tree by $\mathrm{k}^{\text {th }}$ ant and $G_{k}$ denotes the subgraph generated by $k^{\text {th }}$ ant. Here $G$ denotes the general subgraph and $\mathrm{C}$ denotes the set of candidate edges and $\mathrm{E}$ denotes the set of all the edges in the original structure. Parameter $S^{\mathrm{lb}}$ denotes the local best solution for local search and the parameter $S^{\text {gb }}$ denotes the overall best found structure and $F(S)$ denotes the objective value of the structure or solution S. Every ant starts from the home page node. First, a spaning tree is created by an ant. The spanning tree satisfies the outdegree constraint. Then, other edges are added to generate a subgraph. Then local search is applied to improve the solution generated by the ant. The global pheromone updation is performed to exploit the highly traversed edges.

\section{F. Stopping criteria}

The maximum number of iterations for the main loop is set to 1000 . The termination condition in local search is the maximum number of iterations which is set at $0.2 \times n$, here $n$ are the number of nodes in the graph. If there are hundered nodes then local search run for 20 times everytime to improve the solution. The algorithm ends when one of the following conditions becomes true: the algorithm has run for maximum number of iterations, no improvement has been observed in 100 consecutive iterations.

\section{EXPERIMENTAL RESULTS}

In this section, computational experiments and the numerical results are presented. For evaluating the proposed algorithm, artificial complete web graphs of 50, 100 and 200 nodes were generated. The link access frequencies of random numbers lying between 0 and 1 were assigned to every link of each graph. For the graphs of 50 nodes, the edges of the largest 200, 300, 400 frequencies were selected to form test webgraphs. For the graphs of 100 and 200 nodes, the edges of the largest 300 , 500, 800 and 100, 1500, 2000 frequencies were selected to form more test webgraphs. Then the access frequencies of each test webgraph are normalized. However, the test graphs were modified to make sure that every node has at least one incoming link so that the link structure remains connected and feasible solutions are produced. The outdegree was set to 8 and level was set to 8 and 6. The performance of the algorithm depends significantly on the values of the parameters $\alpha$ (global evaporation rate), $\rho$ (local pheromone evaporation rate), $\mathrm{q}_{0}, \mathrm{~m}, \tau_{0}$. The values of the parameters used in the experiment are $\alpha=0.9, \rho=0.5, \mathrm{q}_{0}=0.5, \mathrm{~m}=7$. The initial pheromone level $\tau_{0}$ was set at 0.1 . Then the proposed optimization model is applied on these web graphs to analyse if the model improves the link structure. The proposed hybrid model is compared with Ant Colony method[11]. Microsoft Visual Studio 2010 and C\# language have been used to develop the algorithms. The experiments have been performed on a personal computer with Pentium i3 $2.5 \mathrm{GHz}$ processor, $4 \mathrm{~GB}$ RAM and Microsoft Windows 7 ultimate operating system. Table 1 shows the comparison of the proposed model with Ant Colony System based model. The objective values shown in the table 1 are the averages of 30 trials. It is being observed that the objective function generated by the ACS degrades as the number of links increases but in the proposed method the degradation is very less because the objective function produced by ant colony method is further improved by applying the local search in which the links having low weights are replaced by higher weight links which improves the objective value. Table 1 summarizes the computational results and comparison of the proposed algorithm with simple ant colony method. It is observed that the proposed hybrid algorithm always perform better than the ant colony method.

Table 1. Comparison between the ACS and proposed ACS with local search method

\begin{tabular}{|c|c|c|c|c|c|}
\hline \multirow[t]{2}{*}{ Nodes } & \multirow[t]{2}{*}{ links } & \multicolumn{2}{|c|}{ Constraints } & \multirow{2}{*}{$\frac{\text { ACS }}{\text { Objective }}$} & \multirow{2}{*}{$\begin{array}{c}\text { ACS with Local search } \\
\text { Objective }\end{array}$} \\
\hline & & Outdegree & Depth & & \\
\hline \multirow{6}{*}{50} & \multirow{2}{*}{200} & 8 & 8 & 0.907 & 0.944 \\
\hline & & 8 & 6 & 0.903 & 0.941 \\
\hline & \multirow{2}{*}{300} & 8 & 8 & 0.855 & 0.913 \\
\hline & & 8 & 6 & 0.851 & 0.909 \\
\hline & \multirow{2}{*}{400} & 8 & 8 & 0.816 & 0.874 \\
\hline & & 8 & 6 & 0.808 & 0.868 \\
\hline \multirow{6}{*}{100} & \multirow{2}{*}{300} & 8 & 8 & 0.913 & 0.948 \\
\hline & & 8 & 6 & 0.905 & 0.939 \\
\hline & \multirow{2}{*}{500} & 8 & 8 & 0.883 & 0.926 \\
\hline & & 8 & 6 & 0.879 & 0.922 \\
\hline & \multirow{2}{*}{800} & 8 & 8 & 0.852 & 0.879 \\
\hline & & 8 & 6 & 0.851 & 0.871 \\
\hline \multirow{6}{*}{200} & \multirow{2}{*}{1000} & 8 & 8 & 0.816 & 0.873 \\
\hline & & 8 & 6 & 0.811 & 0.867 \\
\hline & \multirow{2}{*}{1500} & 8 & 8 & 0.798 & 0.856 \\
\hline & & 8 & 6 & 0.798 & 0.842 \\
\hline & \multirow{2}{*}{2000} & 8 & 8 & 0.752 & 0.821 \\
\hline & & 8 & 6 & 0.741 & 0.808 \\
\hline
\end{tabular}




\section{CONCLUSIONS AND FUTURE WORK}

This paper presents a hybrid algorithm based on Ant colony System and local search for website optimization. The proposed method is compared with the simple Ant colony based method using artificially generated web graphs and it is observed that the developed method always gives higher value of the objective function. Website Structure Optimization problem is very much an unexplored problem. In future work, the presented model would be applied on very large sized webgraphs and also on real world website structures to further analyze its behavior. Future work also includes the use of other metaheuristics to develop new algorithms for website structure.

\section{REFERENCES}

[1] M. Chen, and Y.U. Ryu, "Facilitating effective user navigation through web site structure improvement," IEEE Transactions on Knowledge and Data Engineering, pp. 118, 2013.

[2] A. Colorni, M. Dorigo, and V. Maniezzo, "Distributed optimization by ant colonies," In Toward a practice of autonomous systems: Proceedings of the first European conference on artificial life, Cambridge. MA: MIT Press, pp. 134-142, 1992.

[3] M. Dorigo, and C. Blum, "Ant colony optimization theory: A survey," Theoretical Computer Science, 344, 243-278, 2005.

[4] X. Fang, and C. Holsapple, “ An Empirical Study of Web Site Navigation Structures: Impacts on Web Site Usability. Decision Support Systems," vol. 43, no. 2, pp. 476-491, 2007.

[5] Y. Fu, M. Y. Shih, M. Creado and C. Ju, "Reorganizing web sites based on user access patterns," International Journal of Intelligent Systems on Accounting, Finance and Management. 11, 39-53, 2002.

[6] F. Glover, "Tabu search - Part I," ORSA Journal on Computing 1, 190-206, 1989.

[7] J. Hsu, Data mining trends and developments: the key data mining technologies and applications for the 21 st century, in: ISECON, 2002

[8] W. Kim, Y.U. Song, and J.S. Hong, "Web enabled expert systems using hyperlink-based inference," Expert Systems with Applications, 1-13, 2004.

[9] X-Y Li, P. Tian, and SCH. Leung, "An ant colony optimization metaheuristic hybridized with tabu search for pen vehicle routing problems," Journal of the Operational Research Society, 60, 1012- 1025, 2009.

[10] C. C. Lin, "Optimal web site reorganization considering information overload and search depth," European Journal of Operational Research, 173, pp. 839-848., 2006

[11] C.C. Lin, and L.C. Tseng, "Website reorganization using an ant colony system," Expert Systems with Applications, 37, 7598-7605, 2010.

[12] E.M. Loiola, et al., "A survey for the quadratic assignment problem," European Journal of Operational Research, 176 (2), 657-690, 2007.

[13] J. Palmer, "Web Site Usability, Design, and Performance Metrics," Information Systems Research, vol. 13, no. 2, pp. 151-167, 2002.
[14] M. Perkowitz and O. Etzioni, "Adaptive Web sites: An AI challenge," In IJCA: Proceedings of International Joint Conference on Artificial Intelligence, Nagoya, Japan, pp. 16-21, Morgan Kaufmann, 1997.

[15] M. Perkowitz, and O. Etzioni, "Toward adaptive Web sites: Conceptual framework and case study," Artificial Intelligence, 118, 245-275, 2000.

[16] J. Ramanujam, and P. Sadayappan, "Mapping combinatorial optimization problems onto neural networks," Inf. Sci., vol. 82, no. 3-4, pp. 239-255, 1995.

[17] J. Song, and F.M. Zahedi, "A Theoretical Approach to Web Design in E-Commerce: A Belief Reinforcement Model," Management Science, vol. 51, no. 8, pp. 12191235, 2006.

[18] H.Q. Saremi, B. Abedin, and A.M. Kermani, "Website structure improvement: quadratic assignment problem approach and ant colony meta-heuristic technique," Applied Mathematics and Computation, 195, 285-298, 2008.

[19] J. Srivastava, R. Cooley, M. Deshpande and P. N. Tan, "Web usage mining: discovery and applications of usage patterns from Web data," ACM SIGKDD Explorations Newsletter. Volume 1 Issue 2, Pages 12-23, 2002.

[20] T. Stutzle and H. Hoos, "MAX-MIN Ant System and local search for the traveling salesman problem," IEEE International Conference on Evolutionary Computation, pp. 309-314, 1997.

[21] V. Venkatesh, and R. Agarwal, "From Visitors into Customers: A Usability-Centric Perspective on Purchase Behavior in Electronic Channels," Management Science, vol. 52, no. 3, pp. 367-382, 2006.

[22] Y. Wang, D. Wang, and W. Ip, "Optimal design of link structure for e-supermarket website," IEEE Transactions: Systems, Man and Cybernetics- Part A, 36:338-355, 2006.

[23] P. Yin and Y. Guo, "Optimization of melti-criteria website structure based on enhanced tabu search and web usage mining," Journal of Applied Mathematics and Computation, 219: 11082-11095, 2013.

[24] A. Kaur and D. Dani, "The Navigability Structure of EBanking in India," I.J. Information Technology and Computer Science, 05, 29-37, 2013.

\section{Authors' Profiles}

Harpreet Singh is a $\mathrm{PhD}$ student in the department of Computer Science \& Engg. at Guru Nanak Dev University, Amritsar, India. His research interests include data mining, discrete structures and algorithm analysis.

Parminder Kaur is an Assistant professor in the department of Computer Science \& Engg. at Guru Nanak Dev University, Amritsar, India. She completed her PhD from Guru Nanak Dev University, Amritsar in 2010. Her research interests include data mining, software engineering and metaheuristics. 